\title{
Mobile Phone Based RIMS for Traffic Control a Case Study of Tanzania
}

\author{
Angela-Aida Karugila Runyoro ${ }^{1}$ \\ Technology, Tanzania \\ Email: runyoroa@nm-aist.ac.tz \\ Jesuk Ko ${ }^{2}$ \\ ${ }^{2}$ Department of Healthcare Management, Gwangju University, Korea \\ Email: jko@gwangju.ac.kr
}

${ }^{1}$ Department of Information Communication Science and Engineering, Nelson Mandela African Institute of Science and

\begin{abstract}
Vehicles saturation in transportation infrastructure causes traffic congestion, accidents, transportation delays and environment pollution. This problem can be resolved with proper management of traffic flow. Existing traffic management systems are challenged on capturing and processing real-time road data from wide area road networks. The main purpose of this study is to address the gap by implementing a mobile phone based Road Information Management System. The proposed system integrates three modules for data collection, storage and information dissemination. The modules works together to enable real-time traffic control. Disseminated information from the system, enables road users to adjust their travelling habit, also it allows the traffic lights to control the traffic in relation to the real-time situation occurring on the road. In this paper the system implementation and testing was performed. The results indicated that there is a possibility to track traffic data using Global Positioning System enabled mobile phones, and after processing the collected data, real-time traffic status was displayed on web interface. This enabled road users to know in advance the situation occurring on the roads and hence make proper travelling decision. Further research should consider adjusting the traffic lights control system to understand the disseminated real-time traffic information.
\end{abstract}

Index Terms - Road Information Management System, Mobile Phone Application, Road Traffic Congestion, Software Development, Traffic Management

\section{INTRODUCTION}

System testing and implementation is among the project stages where theoretical design is turned into a working system $[1,2]$. It is built on the results of all prior Software Development Life Circle (SDLC) stages. In this stage components of the system constructed during design and development stage are installed in the production environment. In this research a mobile based Road Information Management System (RIMS) was developed and implemented, purposely for mitigating traffic congestion. W-model was used as a SDLC model for developing and implementing the system. This model was preferred over other models due to its flexibility in implementing systems comprising sub systems. The Wmodel incorporates testing into entire SDLC phases. The model clearly illustrates that testing should start at the very beginning of project. It is claimed in the literature [3] that testing is an optimal way to produce a quality software application. A comprehensive testing management examines all components associated with the application. Even more, testing provides an opportunity to validate and verify things like the assumptions that went into the requirements, the appropriateness of the systems that the application is to run on. More likely though, in testing the focus is mainly on the functionality and reliability of application itself [4]

Reference [5] argues that, implementation phase continues until the system is operating in production in accordance with the defined user requirements. Testing is involved in every stage of software life cycle, but the testing done at each level of software development is different in nature and has different objectives. Reference [2] lists items in testing spectrum as follows: - (i) Unit testing is done at the lowest level. It tests the basic unit of software, which is the smallest testable piece of software, and is often called "unit", "module", or "component" interchangeably. (ii) Integration testing is performed when two or more tested units are combined into a larger structure. The test is often done on both the interfaces between the components and the larger structure being constructed, if its quality property cannot be assessed from its components. (iii) System testing tends to affirm the end-to-end quality of the entire system. System test is often based on the functional/requirement specification of the system. Non-functional quality attributes, such as reliability, security, and maintainability, are also checked. (iv) Acceptance testing is done when the completed system is handed over from the developers to the customers or users. The purpose of acceptance testing is rather to give confidence that the system is working than to find errors. Reference [6] recommends that system testing should continue even after its implementation, so as to reduce number of errors which are already present in the system.

Implementation of Road Information Management System (RIMS) incorporated all functionalities of the modules which were developed as its subsystems. The main goal of implementing RIMS in this research is to show that, with a huge number of mobile phones moving all the time across the roads, capturing road information 
on a wide area road network is possible. This will give possibilities of eliminating traffic congestion problem in the studied area (i.e. Dar es Salaam, Tanzania) through real-time road information dissemination [7]. Before starting their trips, road users are provided with the traffic situation information so that they get to know in advance the road situation. Disseminated information alert the road users about existence of traffic congestion on a specific road segment, so that they can find alternatives such as: - (i) Choose alternative roads to the destination, and skip the jam. (ii) Use other means of transportation such as, instead of driving a private car they can use either public transportation or ride a bicycle. (iii) Change time of travelling, by travelling at a time when there is no congestion.

RIMS were developed by integrating three different sub systems, these are: - (i) Mobile application (ii) Relational Data Base Management System (RDBMS), and (iii) Web site. The mobile application was implemented on Android platform, data storage and processing was implemented in MYSQL RDBMS. Personal Hypertext Pre-processor (PHP), Java Script and Hypertext Markup Language (HTML) were used to implement the website on Apache web server. The system implementation started by installing the mobile application clients, these are sub systems which have main responsibility of road data collection. The sub system is considered as source of information to all other subsystems in RIMS. References [8, 9] argue that mobile phones are diffusing rapidly in developing countries but research conceptualizations have been lagging behind practice, particularly those that link mobile phones to livelihoods. To fill the gap of proper utilization of mobile phones to solve problems of the poor, where traffic congestion is among the problems, this research has explained in details the system testing and implementation procedures that can be followed to mitigate the traffic congestion problem using a mobile phone based RIMS. We have discovered that road information can be easily collected using mobile phones which are all the time available on the roads. The collected road data then gets processed and stored in a database, at the end the web interface is used to disseminate traffic information to the road users. In order to solve congestion problem there have been several ideas, most of them are suggesting road information sharing [10]. Floating Car Data (FCD) is one of the methods used to gather traffic information [7]. It uses vehicles as sensor nodes that transmit their speed to the server. The server then broadcasts the updated speed data to all vehicles on the road so that vehicles can compute optimal travel routes based on current traffic data [11]. Hence road information management conducted in this research generates the probability to create more intelligent roads, vehicles and users. This leads to congestion mitigation through information sharing.

This paper is organized as follows. Section 2 introduces the overall approach to solve the traffic congestion problem. Section 3 illustrates the application of the proposed system, RIMS in realistic traffic test cases, and describes the research findings. Section 4 draws conclusion of this study.

\section{METHODOLOGY}

The initial focus of research was made on design and development of RIMS. After the development, then RIMS was tested and implemented purposely to solve the road traffic congestion problem which is currently affecting the daily life of Tanzanians. In order to test and implement the system, the developed modules were installed on hardware and system performance on real situation was monitored.

\section{A. System Installation and Testing}

System testing was conducted in Dar es Salaam city. Five smart phones installed with the road data collection application were distributed to five bus commuters' drivers (daladala drivers). Three bus routes were selected for testing, these are: - (i) Bagamoyo road (Mwenge salender bridge) (ii) Morogoro road (Ubungo - Akiba) (iii) Nelson Mandela road (Ubungo - Tazara). Figure 1 shows the road segments considered for system testing. The mobile phones were distributed in a way that, each road to have at least two phones collecting the data. Two phones were used for Bagamoyo road, the other two phones were used for Morogoro road and one phone was used for Nelson Mandela road. The participating drivers were guided to start the mobile application (tztraffman Tanzania Traffic Manager) whenever they were travelling on the selected road segments. This enabled the mobile phones to collect and send data from only the intended locations. Test cases to check the mobile application performance were created.

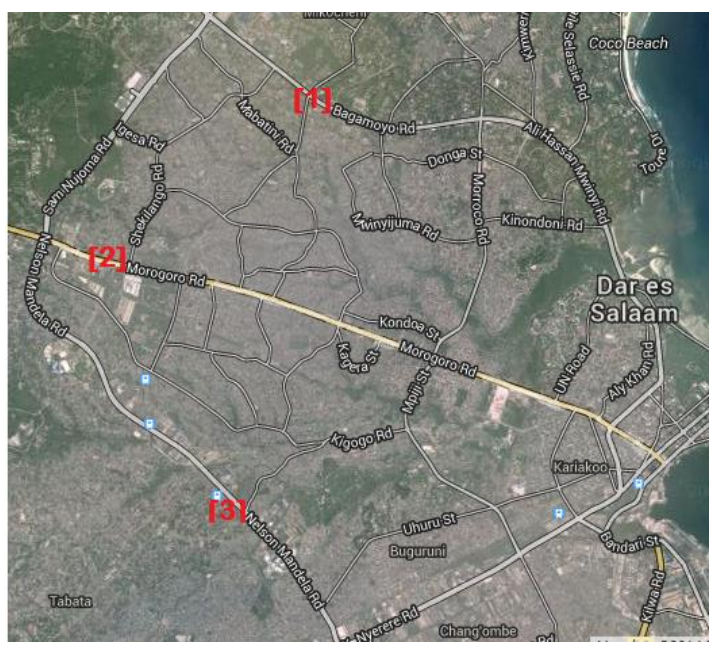

Fig. 1. Road Segments for System Testing

\section{B. Data Storage and Processing}

From the mobile application, road data was posted to the database. The mobile application was configured to send data after every ten seconds. The data included phone's International Mobile Equipment Identity (IMEI) number, latitude and longitude coordinates and speed. Test cases to check the collected data in the database were created. 


\section{Road Traffic Information Dissemination}

Web interface which considers responsive design was created, the interface has capability of being accessed using both computers and mobile devices (tablets, mobile phones) [12]. Using PHP scripts the collected road data were processed to find the real-time traffic condition. The system was configured to calculate average speed from the recorded speed of the past twenty seconds from the time when the road user sends an information request. This was done purposely to capture the real-time traffic condition. The interface provides a form which is used by the road users to send a request to the server. The request includes specific road segments needed by the user. The request is then processed in relation to the selected road segment. Real-time road traffic condition of the selected segment is displayed as a feedback message to the road user. From the message, the road users are free to make a proper travelling decision in relation to the present traffic situation.

\section{EXPERIMENTAL RESULTS}

\section{A. System Setup and Execution}

RIMS was installed comprising three modules, the first module is road data collection mobile application. Figure 2, 3 and 4 show the screen shots of the module. When the system starts, the first screen Figure 2 is displayed to give introductory information of the system. It guides the drivers to start the application whenever they are driving. This enables the phone to collect data while on the road. Figure 3 shows the configuration screen, the driver has to enter appropriate phone's IMEI number, so as it can be easily recognised by the server. The server Internet Protocol (IP) address together with the port number are needed in the mobile application, to make sure that the device is going to send road data to that specific server. The last part of the mobile application is shown on Figure 4 , where connection to the server success or failure message is displayed. Whenever the mobile application sends the road data, a location update message is displayed.

Screen shots for the data collection module:

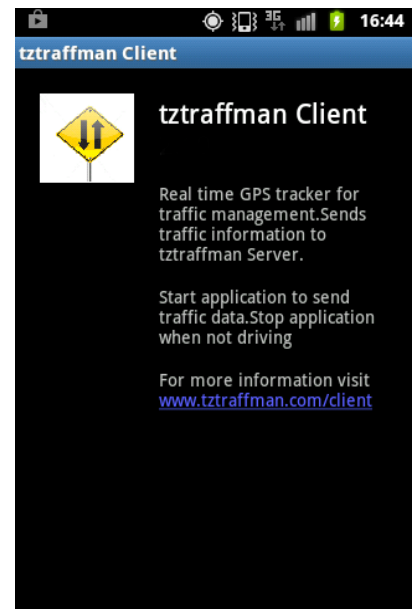

Fig. 2. Mobile Application Home Screen

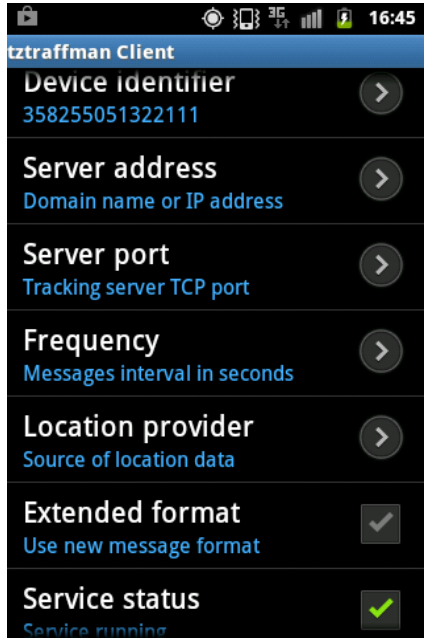

Fig. 3. Mobile Application Configuration Screen

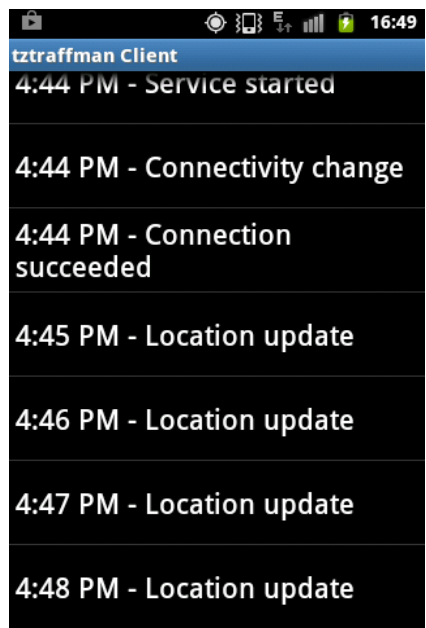

Fig. 4. Mobile Application Road Data Collection Screen

\section{Screen shot for the data storage and processing:}

The second module is data storage and processing server. All road data collected by the mobile application is stored in the database. The stored data can be viewed by browsing the specific tables in the database as shown on Figure 5.

\section{Screen shots for the information dissemination interface:}

The last module is traffic information dissemination interfaces. Figure 6 and 7 show screen shot of the interfaces. The road users send a request for real-time traffic information of a specific road segment. Figure 6 shows the form utilized by users to select both start and destination point road junctions. From these data the system can easily figure out a specific road segment needed. Feedback message indicating traffic situation of the selected road segment is then displayed as shown on Figure 7. By processing the collected road data together with the posted request, the system can determine whether there is traffic congestion, slow motion or free flow on a specific road segment. 




Fig. 5. Stored Road Data

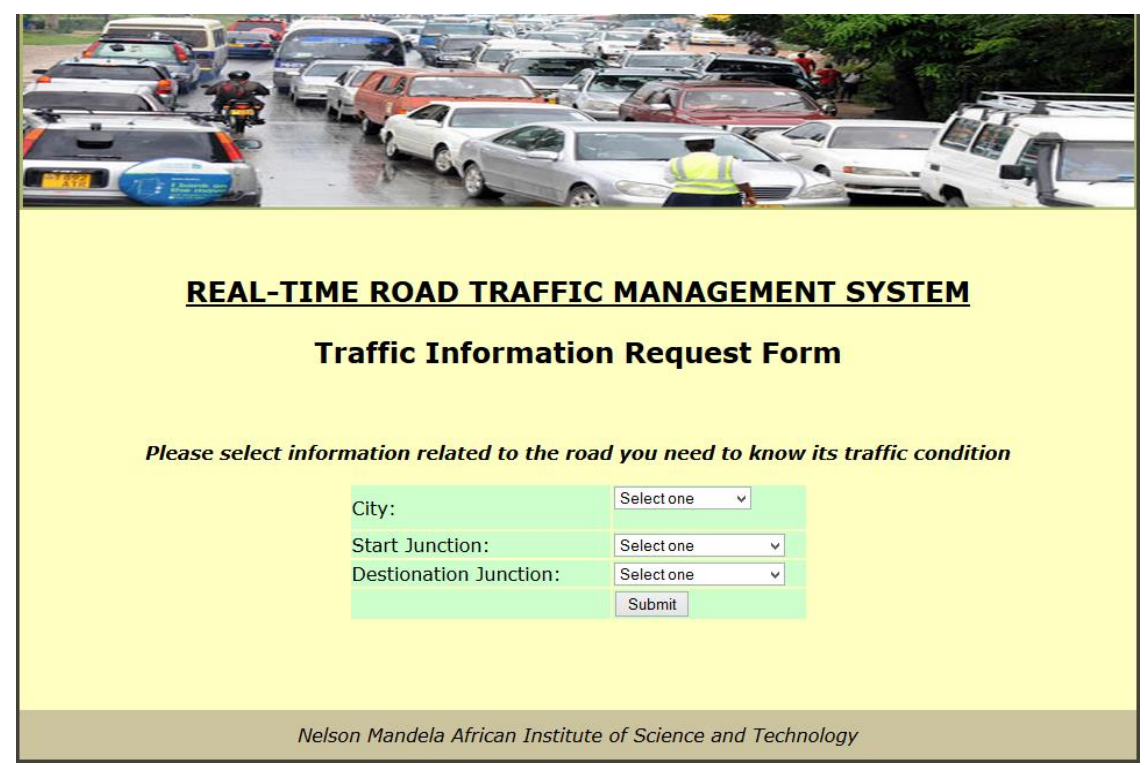

Fig. 6. Traffic Information Request Interface

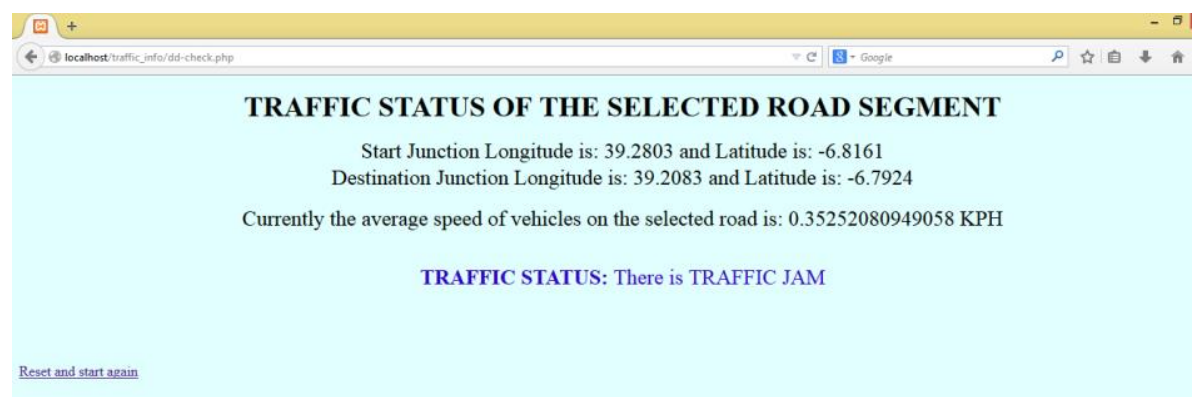

Fig. 7. Traffic Information Retrieve Interface 


\section{B. System Testing}

System testing is one of the "verification and validation software practices [13]. Through verification, we make sure the product behaves the way we want it to, and Through validation, we check to make sure that somewhere in the process a mistake hasn't been made; validation always involves comparison against requirements [14]. There are two basic classes of software testing, black box testing and white box testing [15].

In this paper implementation of black box test on RIMS is presented. The choice of test cases for functional testing is based on the requirement or design specification of the RIMS, Table 1 lists all the test cases. Expected results or test oracles, included requirement/design specifications, hand calculated values, and simulated results. Functional testing mainly focus on external behaviour of the software entity [6], this was checked from known inputs against expected results.

As indicated in Table 1, functional and behaviour of RIMS were tested through determining whether or not the system does what it is supposed to do. The black box testing was considered, since the application codes and logic structure of the application were already tested during system development phase.

During the testing activity, test cases were generated to find circumstances where the application does not respond as required and revealing bugs or defects. Test cases were written to cover things that the user will do most often. Our objective was to find as many defects as possible using as few test cases as possible.

Table 1. RIMS Testing

\begin{tabular}{|c|c|c|c|c|c|}
\hline $\begin{array}{c}\text { Test Case } \\
\text { ID }\end{array}$ & Description & Expected Results & Actual Results & $\begin{array}{l}\text { Pass } \\
\text { / Fail }\end{array}$ & Comments \\
\hline 1 & \multicolumn{5}{|c|}{ Precondition: Mobile phone is switched on } \\
\hline & $\begin{array}{l}\text { Application icon } \\
\text { clicked to start } \\
\text { road data } \\
\text { collection mobile } \\
\text { application }\end{array}$ & $\begin{array}{c}\text { Mobile application home } \\
\text { page displayed }\end{array}$ & $\begin{array}{c}\text { Mobile application home } \\
\text { page displayed }\end{array}$ & Pass & \\
\hline \multirow[t]{2}{*}{2} & \multicolumn{5}{|c|}{ Precondition: GPS service enabled } \\
\hline & $\begin{array}{c}\text { Do mobile } \\
\text { application setup, } \\
\text { enter IMEI } \\
\text { number, server IP } \\
\text { address and port } \\
\text { number on } \\
\text { configuration } \\
\text { screen }\end{array}$ & $\begin{array}{l}\text { - Correct data entered: Service } \\
\text { starting, connections to the } \\
\text { server succeed, road data are } \\
\text { updated and location update } \\
\text { message displayed on road data } \\
\text { collection screen } \\
\text { - Wrong data entered: } \\
\text { Connection failure message is } \\
\text { displayed }\end{array}$ & $\begin{array}{l}\text { - With correct data entered: } \\
\text { Connection to the server } \\
\text { succeeded and road data } \\
\text { were updated } \\
\text { - With wrong data entered: } \\
\text { No response was displayed }\end{array}$ & Fail & $\begin{array}{l}\text { With wrong data entered } \\
\text { nothing was displayed, } \\
\text { bug fixing was done to } \\
\text { display connection failure } \\
\text { message }\end{array}$ \\
\hline \multirow[t]{2}{*}{3} & \multicolumn{5}{|c|}{ Precondition: Phone registration in the database, IMEI number recorded } \\
\hline & & $\begin{array}{c}\text { Road data collected by } \\
\text { registered phones are updated in } \\
\text { the database after every } 10 \\
\text { seconds }\end{array}$ & $\begin{array}{l}\text { Road data are updated in the } \\
\text { database after every } 10 \\
\text { seconds }\end{array}$ & Pass & $\begin{array}{l}10 \text { seconds was set as the } \\
\text { minimum time interval to } \\
\text { capture real-time changes }\end{array}$ \\
\hline \multirow[t]{4}{*}{4} & \multicolumn{5}{|c|}{ Precondition: URL for the road information request web page entered in the browser for opening } \\
\hline & & $\begin{array}{l}\text { Road information request form } \\
\text { displayed }\end{array}$ & & Pass & \\
\hline & & $\begin{array}{l}\text { Start and end junctions for the } \\
\text { intended road segments are } \\
\text { selected, request is submitted by } \\
\text { clicking submit button }\end{array}$ & $\begin{array}{l}\text { Start and end junctions for } \\
\text { the intended road segments } \\
\text { are selected, request is } \\
\text { submitted by clicking } \\
\text { submit button }\end{array}$ & Pass & \\
\hline & & $\begin{array}{l}\text { Real-time road traffic status of } \\
\text { the selected road segment is } \\
\text { displayed on the new page }\end{array}$ & $\begin{array}{l}\text { Traffic status of the past } \\
\text { 1minute is displayed }\end{array}$ & Fail & $\begin{array}{l}\text { Time adjustment was done } \\
\text { to calculate average speed } \\
\text { for the past } 20 \text { seconds, } \\
\text { this approximate to real- } \\
\text { time }\end{array}$ \\
\hline
\end{tabular}

\section{CONCLUSION}

It has been noted that, when the implementation phase concludes, the system begins operating and continues to do so until the organization determines it has outlived its usefulness and starts planning for a replacement system. The approval of the implementation phase deliverables and the completion of the implementation project status review, and the execution of project close-out activities, signify the end of the implementation Phase. RIMS design and development work considered planning and analysis of the system. RIMS modules were integrated to enable road information management which leads all activities done in the system to traffic control. 
In the future, system development should also consider other mobile platforms different from Android, such as Microsoft Windows, Symbian OS and iOS [16]. This will enable varieties of mobile phones to be used in road data collection. This research has only concentrated on Android platform due to time constraints, and the only available mobile phones for the research were Android based. Big data techniques also should be considered in data storage and processing. Sending road data to the server requires utilization of data transfer service from mobile subscription company, this is costly. Therefore more research is needed to provide a more cost effective solution for road information management.

Currently traffic management in Tanzania is only controlled by traffic lights, more research is needed to integrate the proposed RIMS with the existing traffic light systems, and this will enable the traffic lights to act according to the realistic situation occurring on the road. In the current stage, the traffic light system has no ability to adjust themselves in relation to the occurring situations on the road. Instead they continue to function normally even if there changes on the road such as accident, parades and any other activities which cause traffic jam.

\section{REFERENCES}

[1] Vaughan, P.J. System Implementation Success Factors; It's Not Just the Technology. University of Colorado at Boulder. 2001; Available at the website: https:// net.educause.edu/ ir/library/pdf/CMR0122.pdf Accessed on 10th May 2014.

[2] Luo, L., Software Testing Techniques. Institute for software research international Carnegie mellon university Pittsburgh, PA, 2001. 15232(1-19): p. 19.

[3] Kosindrdecha, N. and J. Daengdej, A test case generation process and technique. J. Software Eng, 2010. 4: p. 265287.

[4] Jenkins, N., A Software Testing Primer, in An Introduction to Software Testing. 2008, Creative Commons.

[5] Erdil, K., et al., Software Maintenance as Part of the Software Life Cycle. Comp180: Software Engineering Project, 2003.

[6] Jenkins, N., A Software Testing Primer. An Introduction to Software Testing, 2008.

[7] Runyoro, A.-A.K. and J. Ko, Real-Time Road Traffic Management Using Floating Car Data. International Journal of Fuzzy Logic and Intelligent Systems, 2013. 13(4): p. 269-276.

[8] Duncombe, R., Researching Impact of Mobile Phones for Development: Concepts, Methods and Lessons for Practice. Information Technology for Development, 2011. 17(4): p. 268-288.

[9] Duncombe, R. Understanding Mobile Phone Impact on Livelihoods in Developing Countries: A new Research Framework. Manchester Centre for Development Informatics, Working Paper 48, 2012; Available at the website:

http:// www.sed.manchester.ac.uk/idpm/research/publications/wp /di/di_wp48.htm Accessed on 15th August 2014.

[10] Figueiredo, L., J.T. Machado, and J.R. Ferreira, Dynamical Analysis of Freeway Traffic. IEEE Transactions on Intelligent Transportation Systems, 2004. 5(4): p. 259-266.

[11] Ayala, D., et al. Communication Reduction for Floating Car Data-Based Traffic Information Systems. In
Proceedings of IEEE Second International Conference on Advanced Geographic Information Systems, Applications, and Services (GEOPROCESSING), 2010.

[12] Anam, R., C.K. Ho, and T.Y. Lim, Tree Adapt: Web Content Adaptation for Mobile Devices. International Journal of Information Technology and Computer Science (IJITCS), 2014. 6(9): p. 1-13.

[13] Zheng, J., et al., On the Value of Static Analysis for Fault Detection in Software. Software Engineering, IEEE Transactions on, 2006. 32(4): p. 240-253.

[14] Rakitin, S.R., Software Verification and Validation for Practitioners and Managers. 2001: Artech House, Inc.

[15] Nidhra, S. and J. Dondeti, Black Box and White Box Testing Techniques - A Literature Review. International Journal of Embedded Systems and Applications (IJESA), 2012. 2(2): p. 29-50.

[16] Godwin-Jones, R., Emerging Technologies: Mobile Apps for Language Learning. Language Learning \& Technology, 2011. 15(2): p. 2-11.

\section{Authors' Profiles}

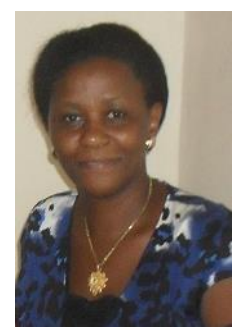

Dr. Angela-Aida K. Runyoro obtained her Ph.D. degree in the School of Computational and Communications Science and Engineering at the Nelson Mandela African Institute of Science and Technology (NM-AIST), Arusha in Tanzania. She received her Postgraduate Diploma in Managerial Control and Management Information Systems from the Maastricht School of Management in the Netherlands. She holds a Master's degree in Computer Science and Bachelor degree in Electrical Engineering from the University of Dar es salaam in Tanzania. She also received an advanced Certificate in Information Technology for African Business from Inwent in German. Her main research interests are systems analysis and design, intelligent systems development, and integrated traffic management.

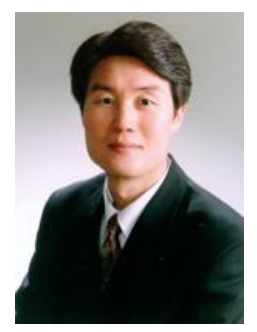

Prof. Jesuk Ko received his $\mathrm{PhD}$ degree in Systems Engineering from Royal Melbourne University of Technology (RMIT) in Australia, and his MSc. degree in Industrial Engineering from Pohang University of Science and Technology $(\mathrm{POSTECH})$ in Korea, respectively. As a Professor, he is currently Head of the Healthcare Management Department at Gwangju University. Also he is an Adjunct Professor of the Nelson Mandela African Institute of Science and Technology (NM-AIST) in Tanzania. He was a Visiting Scholar in the Department of Engineering at the Australian National University (ANU) for the period of 2008 to 2009. He was also Head of the Industrial and Information Engineering Department at Gwangju University. He served as a Full-time Lecturer in the Graduate School of Engineering (GSE) at RMIT. He has been involved in Paper Review Committees as an Editorial Board Member of the prestigious international journals such as JHM, AJOR, APMR, IJPE and others. His research interests include intelligent systems development, AI applications in decision analysis, integrated traffic management and ITS, etc. 